\title{
FORMAL SOLUTION OF THE MASTER EQUATION VIA HPT AND DEFORMATION THEORY
}

\author{
Johannes Huebschmann and Jim StashefF \\ USTL, UFR de Mathématiques, \\ Math-UNC
}

\begin{abstract}
We construct a solution of the master equation by means of standard tools from homological perturbation theory under just the hypothesis that the ground field be of characteristic zero, thereby avoiding the formality assumption of the relevant dg Lie algebra. To this end, we endow the homology $\mathrm{H}(\mathfrak{g})$ of any differential graded Lie algebra $\mathfrak{g}$ over a field of characteristic zero with an sh-Lie structure such that $\mathfrak{g}$ and $\mathrm{H}(\mathfrak{g})$ are sh-equivalent. We discuss our solution of the master equation in the context of deformation theory. Given the extra structure appropriate to the extended moduli space of complex structures on a Calabi-Yau manifold, the known solutions result as a special case.
\end{abstract}

1991 Mathematics Subject Classification. 13D10 14J32 16S80 16W30 17B55 17B56 17B65 17B66 17B70 17B81 18G10 32G05 55P62 55R15 81T70.

Key words and phrases. Twisting cochain, deformation equation, master equation, classification of rational homotopy types, formal power series connection, differential graded algebra, differential graded Lie algebra, sh-Lie algebra, homological perturbation theory, dG algebra, dBV algebra, Frobenius structure. 


\section{Introduction}

The name "Master Equation" derives from the physics literature, especially that of the Batalin-Vilkovisky approach to Lagrangians with symmetries [2], but the master equation has many precursors in mathematics, among which the best known is perhaps the Maurer-Cartan equation. The master equation makes sense as an equation on elements of a differential graded algebra, associative or Lie or suitable higher homotopy generalizations. As an equation on elements of a differential graded Lie algebra $\mathcal{L}$, for an element $\tau$ of $\mathcal{L}$, the master equation has the form

$$
D \tau=\frac{1}{2}[\tau, \tau]
$$

In the literature, it is also customary to take the equation $D \tau+\frac{1}{2}[\tau, \tau]=0$ as (Lie algebra) master equation, the choice of sign being a matter of convention. As an equation on elements of a differential graded associative algebra $\mathcal{A}$, the master equation has the form $D \tau=\tau \tau$. By the methods of homological perturbation theory, we will construct formal solutions of the master equation in the following general context. Consider a differential graded Lie algebra $\mathfrak{g}$, and denote by $\mathcal{S}^{\mathrm{c}}[s \mathfrak{g}]$ the differential graded symmetric coalgebra on the suspension $s \mathfrak{g}$ of $\mathfrak{g}$; then $\operatorname{Hom}\left(\mathcal{S}^{\mathrm{c}}[s \mathfrak{g}], \mathfrak{g}\right)$ inherits a differential graded Lie algebra structure and, in view of the defining property of a Lie algebra twisting cochain (see Section 1 below for details), the universal twisting cochain $\tau_{\mathfrak{g}} \in \operatorname{Hom}\left(\mathcal{S}^{\mathrm{c}}[s \mathfrak{g}], \mathfrak{g}\right)$ satisfies the equation $D \tau_{\mathfrak{g}}=\frac{1}{2}\left[\tau_{\mathfrak{g}}, \tau_{\mathfrak{g}}\right]$ which is plainly just the master equation. By means of our main result, Theorem 2.7 below, up to s(trong) h(omotopy) equivalence, we can then replace the differential graded coalgebra $\mathcal{S}^{\mathrm{c}}[s \mathfrak{g}]$ with a differential graded coalgebra of the kind $\mathcal{S}_{\mathcal{D}}^{\mathrm{c}}[s \mathrm{H}(\mathfrak{g})]$ where $\mathrm{H}(\mathfrak{g})$ is the homology of $\mathfrak{g}$ and $\mathcal{D}$ a coalgebra differential on $\mathcal{S}^{\mathrm{c}}[s \mathrm{H}(\mathfrak{g})]$ turning the latter into a coaugmented differential graded coalgebra - the differential $\mathcal{D}$ defines an sh-Lie structure on $\mathrm{H}(\mathfrak{g})$, see Section 2 below-in such a way that the following hold: The differential graded Lie algebra $\mathcal{L}=\operatorname{Hom}\left(\mathcal{S}_{\mathcal{D}}^{\mathrm{c}}[s \mathrm{H}(\mathfrak{g})], \mathfrak{g}\right)$ is sh-equivalent to $\operatorname{Hom}\left(\mathcal{S}^{\mathrm{c}}[s \mathfrak{g}], \mathfrak{g}\right)$, and the data give rise to a twisting cochain $\tau \in \operatorname{Hom}\left(\mathcal{S}_{\mathcal{D}}^{\mathrm{c}}[s \mathrm{H}(\mathfrak{g})], \mathfrak{g}\right)$ which, in a suitable sense, is equivalent to $\tau_{\mathfrak{g}}$. The twisting cochain $\tau$ is our most general solution of the master equation. Our approach in terms of homological perturbation theory will yield explicit recursive formulas for $\mathcal{D}$ and $\tau$, once a choice of contraction $(\mathrm{H}(\mathfrak{g}) \stackrel{\pi}{\stackrel{\leftrightarrows}{\nabla}} \mathfrak{g}, h)$ of chain complexes has been made. This is always possible over a field. (See Section 2 and especially (2.7.3) and (2.8.2) below for details.) Our Theorem 2.7 also establishes the fact (which has been known to both of us for some time) that, roughly speaking, sh-Lie structures are preserved under strong deformation retractions; in particular, given a differential graded Lie algebra $\mathfrak{g}$, its homology $\mathrm{H}(\mathfrak{g})$ admits an sh-Lie structure such that $\mathfrak{g}$ and $\mathrm{H}(\mathfrak{g})$ are sh-equivalent.

A situation of particular interest recently is that in which $\mathfrak{g}$ is in fact a differential Gerstenhaber algebra. In the case of the moduli space of complex structures on a complex manifold $M$, the relevant differential Gerstenhaber algebra contains the ordinary Kodaira-Spencer (differential graded Lie) algebra which, in turn, is well known to control deformations of the complex structure. When $M$ is a CalabiYau manifold, Barannikov-Kontsevich [1] construct a formal solution of the master equation. They use this solution to construct a formal Frobenius manifold structure 
on the extended moduli space of complex structures on $M$. The BarannikovKontsevich formal solution of the master equation results from our construction as a special case, see Theorem 3.8 below. Thus our homological perturbation theory construction of the twisting cochain $\tau$ extends the known constructions of solutions of the master equation to much less restrictive hypotheses (indeed, over a field of characteristic zero, we do not need a hypothesis at all) and, in particular, allows for generalization to the case in which the underlying manifold does not admit a Kähler structure.

We have already observed that the master equation recovers the defining property of a twisting cochain. Twisting cochains have a history in topology and differential homological algebra $[3,6,41]$. Certain twisting cochains were studied by Chen $[8,9]$ under the name power series connection. Berikashvili and his students have studied the classification of fibrations with fixed base and fiber using Berikashvili's functor $D$ which is defined in terms of homotopy classes of twisting cochains (or twisting elements); see [44] (Section 2) or [31] for a recent account of Berikashvili's functor $D$ and references to work of Berikashvili, Kadeishvili, and Saneblidze in [31, 44]. Twisting cochains play a crucial role in the deformation theory for rational homotopy types and rational fibrations and for the corresponding classification theory developed by Schlessinger and Stasheff in [45], as well as for the construction of small resolutions for doing calculations in group cohomology [22-25].

That twisting cochains may be constructed by means of homological perturbation theory has been known for some time, see e. g. [19], [22] (2.11), [42] (2.2). More historical comments about homological perturbation theory may be found e. g. in Section 1 (p. 248) and Section 2 (p. 261) of [32], which has one of the strongest results in relation to compatibility with other (such as algebra or coalgebra) structure, since it was perhaps first recognized in [22]. Homological perturbation theory constructions enabled the first-named author to carry out complete numerical calculations in group cohomology [23], [24], [25] which cannot be done by other methods. This was an instance where homological perturbation theory led to results which are independent of this theory. The solution $\tau$ of the master equation and its offspring constructed in the present paper constitute as well a result which is phrased independently of homological perturbation theory. The significance of our more general construction for deformation theory is not yet fully understood. It is interesting to observe, though, that the inductive construction of the formal power series $\varphi(t)$ in $\S 3$ of [33] may be seen as a homological perturbation theory construction. Some applications related with [45] and generalizations will be given in Section 4.

\section{Master equation and twisting cochains}

The ground ring is assumed to contain the rationals as a subring and will be written $R$. We will take chain complex or $d g$ module to mean a differential graded $R$-module. A chain complex will not necessarily be concentrated in non-negative (or non-positive) degrees. In the present section and in Section 2, the differential $d$ on a chain complex $M$ will be considered as lowering degree by 1 and referred to as a homology differential (if need be); a cohomology differential $\delta: M^{j} \rightarrow M^{j+1}$ may always be written in this way as $d: M_{-j} \rightarrow M_{-j-1}$ where $M_{*}=M^{-*}$ etc. and a homology differential may accordingly be written as a cohomology differential. We will come back to this in Section 3 below. 
Write $s$ for the suspension operator as usual and accordingly $s^{-1}$ for the desuspension operator. Thus $(s M)_{j}=M_{j-1}$, etc. The algebraic geometer's notation for the suspended (or desuspended, depending on terminology) object is $\mathcal{A}[1]$ or $\mathcal{A}[-1]$ (depending on the convention of grading). We avoid this notation.

The coaugmentation map of a coaugmented differential graded coalgebra $C$ will be denoted by $\eta: R \rightarrow C$. The graded tensor algebra, graded tensor coalgebra, and graded symmetric coalgebra functors will be written respectively $\mathrm{T}, \mathrm{T}^{\mathrm{c}}, \mathcal{S}^{\mathrm{c}}$; see what is said below for details. Given two chain complexes $X$ and $Y$, recall that $\operatorname{Hom}(X, Y)$ inherits the structure of a chain complex by the operator $D$ defined by

$$
D \phi=d \phi-(-1)^{|\phi|} \phi d
$$

where $\phi$ is a homogeneous homomorphism from $X$ to $Y$ and where $|\phi|$ refers to its degree.

Definition 1.1. A $d g$ Lie algebra $\mathfrak{g}$ is a $\operatorname{dg}$ module $\mathfrak{g}=\left\{\mathfrak{g}_{n}\right\}$ with a differential $d: \mathfrak{g}_{n} \rightarrow \mathfrak{g}_{n-1}$ and a graded degree zero bracket [, ]: $\mathfrak{g}_{p} \otimes \mathfrak{g}_{q} \rightarrow \mathfrak{g}_{p+q}$ which is a chain map and satisfies the graded Jacobi identity, that is, for homogeneous $X \in \mathfrak{g}$, the operation $[X$,$] is a graded derivation for the bracket.$

Definition 1.2. For a filtered $\mathrm{dg}$ module $X$, a perturbation of the differential $d$ of $X$ is a (homogeneous) morphism $\partial$ of the same degree as $d$ such that $\partial$ lowers the filtration and $(d+\partial)^{2}=0$ or, equivalently,

$$
[d, \partial]+\partial \partial=0
$$

Thus, when $\partial$ is a perturbation on $X$, the sum $d+\partial$, referred to as the perturbed differential, endows $X$ with a new differential. When $X$ has a graded coalgebra structure such that $(X, d)$ is a differential graded coalgebra, and when the perturbed differential $d+\partial$ is compatible with the graded coalgebra structure, we refer to $\partial$ as a coalgebra perturbation; similarly, we can also talk about an algebra perturbation.

Consider a more 'sophisticated' description of dg Lie algebra: Let $M$ be a chain complex. Write

$$
\mathrm{T}^{\mathrm{c}}[M]=\left(\oplus_{k \geq 0} M^{\otimes k}, \Delta, d\right)
$$

for the coaugmented differential graded tensor coalgebra on $M$; here $\Delta$ and $d$ refer to the diagonal and differential respectively, $M^{\otimes 0}=R$, the coaugmentation map $\eta: R \rightarrow \mathrm{T}^{\mathrm{c}}[M]$ is the canonical one, and, for every $n \geq 0$,

$$
\Delta\left(m_{1} \otimes \cdots \otimes m_{n}\right)=\sum_{p=0}^{n}\left(m_{1} \otimes \cdots \otimes m_{p}\right) \otimes\left(m_{p+1} \otimes \cdots \otimes m_{n}\right),
$$

where $m_{1}, \ldots, m_{n} \in M$. The differential graded symmetric coalgebra $\mathcal{S}^{\mathrm{c}}[M]$ on $M$ has the form

$$
\mathcal{S}^{\mathrm{c}}[M]=\oplus \mathcal{S}_{k}^{\mathrm{c}}[M]
$$

where $\mathcal{S}_{0}^{\mathrm{c}}[M]=R$ and where, for $k \geq 1, \mathcal{S}_{k}^{\mathrm{c}}[M]$ is the differential graded subspace

$$
\mathcal{S}_{k}^{\mathrm{c}}[M]=\left(M^{\otimes k}\right)^{\Sigma_{k}} \subseteq \mathrm{T}_{k}^{\mathrm{c}}[M]=M^{\otimes k}
$$


of invariants under the canonical action (in the graded sense) of the symmetric group $\Sigma_{k}$ on $k$-letters on the $k^{\prime}$ th tensor power $M^{\otimes k}$; see e. g. [26].

Let $\mathfrak{g}$ be a chain complex, endowed with a graded skew-symmetric bracket $[]:, \mathfrak{g} \otimes \mathfrak{g} \rightarrow \mathfrak{g}$ of degree zero, not necessarily a graded Lie bracket nor necessarily a chain map. Consider the differential graded symmetric coalgebra $\mathcal{S}^{\mathrm{c}}[s \mathfrak{g}]$, the differential $d$ on $\mathcal{S}^{\mathrm{c}}[s \mathfrak{g}]$ being induced from that on $\mathfrak{g}$, and let $\partial$ be the coderivation $\mathcal{S}^{\mathrm{c}}[s \mathfrak{g}] \rightarrow \mathcal{S}^{\mathrm{c}}[s \mathfrak{g}]$ of degree -1 induced by [, ]; it is manifest that $\partial$ lowers the obvious filtration of $\mathcal{S}^{\mathrm{c}}[s \mathfrak{g}]$. We then have the following folk-lore result a proof of which is straightforward and left to the reader.

Proposition 1.3. The bracket [, ] turns $\mathfrak{g}$ into a dg Lie algebra if and only if $\partial$ is a coalgebra perturbation of the differential $d$. Furthermore, any differential graded Lie bracket on $\mathfrak{g}$ is determined by the coalgebra perturbation induced from the bracket.

The more 'sophisticated' description of a dg Lie algebra alluded to earlier is that in terms of the coalgebra perturbation $\partial$ given in Proposition 1.3. Below we will abstract from this description and take coalgebra perturbations which do not necessarily correspond to true Lie brackets. Suffice it to explain that, under the circumstances of Proposition 1.3, the vanishing of $(d+\partial)^{2}$ is equivalent to the bracket being a chain map satisfying the graded Jacobi identity. More precisely, the vanishing of $(d+\partial)^{2}$ is equivalent to that of $d \partial+\partial d$ and $\partial \partial$ separately; now $d \partial+\partial d$ to be zero corresponds to the bracket being a chain map, and the vanishing of $\partial \partial$ is equivalent to the graded Jacobi identity. When $\mathfrak{g}$ is an ordinary Lie algebra over a field $\mathbf{k}, \mathcal{S}^{\mathrm{c}}[s \mathfrak{g}]$ with this differential $\partial$ boils down to the ordinary Koszul or Chevalley-Eilenberg complex calculating the homology of $\mathfrak{g}$ with coefficients in $\mathbf{k}$; the $\operatorname{dual} \operatorname{Hom}\left(\mathcal{S}_{[,, \cdot]}^{\mathrm{c}}[s \mathfrak{g}], \mathbf{k}\right)$ is then the Chevalley-Eilenberg algebra calculating the cohomology of $\mathfrak{g}$ with coefficients in $\mathbf{k}$. For a general differential graded Lie algebra $\mathfrak{g}$, with Lie bracket [, ], we will occasionally write $\mathcal{S}_{[,, \cdot]}^{\mathrm{c}}[s \mathfrak{g}]$ for the resulting coaugmented differential graded cocommutative coalgebra or, more simply, following [43] (Appendix) just $\mathcal{C}[\mathfrak{g}]$ and refer to it as its generalized Koszul or Chevalley-Eilenberg complex.

We now explain the Master Equation and the formal circumstances thereof. In general, the master equation makes sense as an equation on elements of a differential graded associative algebra $\mathcal{A}$; it then has the form

$$
D \tau=\tau \tau
$$

where $\tau$ is a homogeneous element of $\mathcal{A}$ (necessarily of degree -1 ), where $D$ refers to the differential in $\mathcal{A}$, and where $\tau \tau$ is the product in $\mathcal{A}$ of $\tau$ with itself. Likewise, as an equation on elements of a differential graded Lie algebra $\mathcal{L}$, for an element $\tau$ of $\mathcal{L}$, the master equation has the form

$$
D \tau=\frac{1}{2}[\tau, \tau] .
$$

When $\mathcal{A}$ is the universal differential graded algebra of a differential graded Lie algebra $\mathcal{L}$, for an element $\tau$ of $\mathcal{L}$, the master equations (1.4.1) and (1.4.2) are 
manifestly equivalent. Inspection shows that, given a differential graded algebra $\mathcal{A}$ and a solution $\tau$ of the master equation, the operator $d_{\tau}$ on $\mathcal{A}$ defined by

$$
d_{\tau}(a)=d a-\tau a, \quad a \in \mathcal{A},
$$

yields a new differential graded algebra structure on $\mathcal{A}$. Likewise, given a differential graded Lie algebra $\mathcal{L}$ and a solution $\tau$ of the master equation, the operator $d_{\tau}$ on $\mathcal{L}$ defined by

$$
d_{\tau}(a)=d a-[\tau, a], \quad a \in \mathcal{L},
$$

yields a new differential graded Lie algebra structure on $\mathcal{L}$.

REMARK. In the literature, instead of (1.4.2), it is also customary to take the equation $D \tau+\frac{1}{2}[\tau, \tau]=0$ as (Lie algebra) master equation. The formula (1.4.4) for the new operator must then accordingly be replaced by $d_{\tau}(a)=d a+[\tau, a]$.

Let $\mathfrak{g}$ be a differential graded Lie algebra. We then have the differential $d$ on $\mathcal{C}[\mathfrak{g}]=\mathcal{S}^{\mathrm{c}}[s \mathfrak{g}]$ induced from that on $\mathfrak{g}$ and, cf. Proposition 1.3, the coalgebra perturbation thereof induced from the graded Lie structure on $\mathfrak{g}$. More general coalgebra perturbations of the differential $d$ on $\mathcal{S}^{\mathrm{c}}[s \mathfrak{g}]$ are related to solutions of a certain master equation as we will explain in Section 2. This master equation involves a differential graded Lie algebra (different from $\mathfrak{g}$ ) of a kind which we now explain. Recall that, for any coaugmented graded coalgebra $C$ and any graded Lie algebra $\mathfrak{h}$, given $a, b: C \rightarrow \mathfrak{h}$, (with a slight abuse of notation) their cup bracket $[a, b]$ is given by the composite

$$
C \stackrel{\Delta}{\longrightarrow} C \otimes C \stackrel{a \otimes b}{\longrightarrow} \mathfrak{h} \otimes \mathfrak{h} \stackrel{[\cdot, \cdot]}{\longrightarrow} \mathfrak{h} .
$$

Now coalgebra perturbations of the differential $d$ on $\mathcal{C}[\mathfrak{g}]=\mathcal{S}^{\mathrm{c}}[s \mathfrak{g}]$ (which is induced from that on $\mathfrak{g}$ ) are related to solutions of the master equation in $\mathcal{L}=\operatorname{Hom}(C, \mathfrak{g})$ where $C$ is an appropriate differential graded cocommutative coalgebra sufficiently closely related to $\mathfrak{g}$ (e. g. $C=\mathcal{S}^{\mathrm{c}}[s \mathfrak{g}]$, endowed with the differential induced from that on $\mathfrak{g})$. Such solutions of the master equation may be described in the language of twisting cochains. This is crucial for our method aimed at constructing solutions of the master equation which we will explain in Section 2 below.

Recall that, given a general differential graded coalgebra $C$ and a general differential graded algebra $A$, for $a, b: C \rightarrow A$, their cup product $a \smile b$ is given by the composite

$$
C \stackrel{\Delta}{\longrightarrow} C \otimes C \stackrel{a \otimes b}{\longrightarrow} A \otimes A \stackrel{\mu}{\longrightarrow} A
$$

where $\Delta$ and $\mu$ denote the structure maps. This cup product turns $\operatorname{Hom}(C, A)$ into a differential graded algebra. Furthermore, a coaugmentation and augmentation map of $C$ and $A$ respectively induce an augmentation map for $\operatorname{Hom}(C, A)$.

Definition 1.5. Given a coaugmented differential graded coalgebra $C$ and an augmented differential graded algebra $A$, a twisting cochain $t: C \rightarrow A$ is a homogeneous morphism of degree -1 whose composites with the coaugmentation and augmentation maps are zero and which satisfies

$$
D t=t \smile t .
$$


Given a coaugmented differential graded cocommutative coalgebra $C$ and a differential graded Lie algebra $\mathfrak{h}$, a Lie algebra twisting cochain $t: C \rightarrow \mathfrak{h}$ is a homogeneous morphism of degree -1 whose composite with the coaugmentation map is zero and which satisfies

$$
D t=\frac{1}{2}[t, t] ;
$$

cf. [41], [43].

In particular, when $A$ is the universal differential graded algebra of a differential graded Lie algebra $\mathfrak{h}$ and when $C$ is graded cocommutative, given a morphism $t: C \rightarrow A$ of degree -1 whose values lie in $\mathfrak{h}$, the defining property (1.5.3) for $t$ being a twisting cochain is equivalent to the defining property (1.5.4) for $t$ being a Lie algebra twisting cochain.

For later reference we recall that, for any differential graded Lie algebra $\mathfrak{h}$, its universal Lie algebra twisting cochain

$$
\tau_{\mathfrak{h}}: \mathcal{C}[\mathfrak{h}]=\mathcal{S}_{[,,]}^{\mathfrak{c}}[s \mathfrak{h}] \rightarrow \mathfrak{h},
$$

cf. e. g. Appendix B of [43], is given by $\tau_{\mathfrak{h}}(s x)=x$ for $x \in \mathfrak{h}$ and $\tau_{\mathfrak{h}}(y)=0$ when $y \in \mathcal{S}_{k}^{\mathrm{c}}[s \mathfrak{h}]$ for $k \neq 1$.

\section{Homological Perturbation Theory (HPT)}

Homological perturbation theory is concerned with transferring various kinds of algebraic structure through a homotopy equivalence. We first describe an essential piece of machinery.

DeFinition 2.1. A contraction

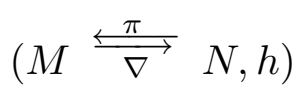

of chain complexes, referred to as well as SDR-data in the literature, consists of

- chain complexes $N$ and $M$,

- chain maps $\pi: N \rightarrow M$ and $\nabla: M \rightarrow N$,

- a morphism $h: N \rightarrow N$ of the underlying graded modules of degree 1;

these data are required to satisfy

$$
\begin{aligned}
\pi \nabla & =\mathrm{Id} \\
D h & =\nabla \pi-\mathrm{Id} \\
\pi h & =0, \quad h \nabla=0, \quad h h=0 .
\end{aligned}
$$

The requirements (2.1.4) are referred to as annihilation properties or side conditions; they can always be achieved without loss of generality, cf. e. g. what is said under 'Remarks' in Section 1 of [15].

Given a contraction (2.1.1), we will say that $N$ contracts onto $M$. If, furthermore, $N$ and $M$ are filtered chain complexes, and if $\pi, \nabla$ and $h$ are filtration preserving, the contraction is said to be filtered. This notion of contraction was introduced in $\S 12$ of [11]; it is among the basic notions in homological perturbation theory, cf. $[18,32]$ and the literature there. 
Let at first $\mathfrak{g}$ be a chain complex, and let

$$
(\mathrm{H}(\mathfrak{g}) \stackrel{\pi}{\stackrel{\nabla}{\leftrightarrows}} \mathfrak{g}, h)
$$

be a contraction of chain complexes. Its existence would, in general, be an additional assumption but such a contraction will always exist when $\mathrm{H}(\mathfrak{g})$ is free over the ground ring $R$ or when $R$ is a field. Under our circumstances, since we assume that the ground ring $R$ contain the rationals as a subring, the existence of a contraction is a very mild assumption, if any. Notice that we momentarily ignore additional structure on $\mathfrak{g}$.

Consider the induced filtered contraction

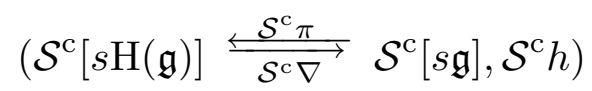

of differential graded coaugmented coalgebras, the filtrations being the ordinary coaugmentation filtrations. It may be obtained in the following way:

Any contraction $(M \stackrel{\vec{\nabla}}{\stackrel{\text { }}{\leftrightarrows}} N, h)$ of chain complexes induces a filtered contraction

$$
\left(\mathrm{T}^{\mathrm{c}}[M] \stackrel{\mathrm{T}^{\mathrm{c}} \pi}{\stackrel{\mathrm{T}^{\mathrm{c}} \nabla}{\rightleftarrows}} \mathrm{T}^{\mathrm{c}}[N], \mathrm{T}^{\mathrm{c}} h\right)
$$

of coaugmented differential graded coalgebras. A version thereof is spelled out as a contraction of bar constructions already in Theorem 12.1 of [11]; the filtered contraction (2.4) may be found in [16] (3.2), [17] (§3), [18] (2.2); the dual filtered contraction of augmented differential graded algebras which involves the differential graded tensor algebra has been given in [32] $(2.2 .0)^{*}$. The differential graded symmetric coalgebras $\mathcal{S}^{\mathrm{c}}[M]$ and $\mathcal{S}^{\mathrm{c}}[N]$ being differential graded subcoalgebras of $\mathrm{T}^{\mathrm{C}}[M]$ and $\mathrm{T}^{\mathrm{C}}[N]$, respectively, the morphisms $\mathrm{T}^{\mathrm{C}} \nabla$ and $\mathrm{T}^{\mathrm{c}} \pi$ pass to corresponding morphisms $\mathcal{S}^{\mathrm{c}} \nabla$ and $\mathcal{S}^{\mathrm{c}} \pi$ respectively, and $\mathcal{S}^{\mathrm{c}} h$ arises from $\mathrm{T}^{\mathrm{c}} h$ by symmetrization, so that

$$
\left(\mathcal{S}^{\mathrm{c}}[M] \stackrel{\stackrel{\mathcal{S}^{\mathrm{c}} \pi}{\leftrightarrows}}{\mathcal{S}^{\mathrm{c}} \nabla} \mathcal{S}^{\mathrm{c}}[N], \mathcal{S}^{\mathrm{c}} h\right)
$$

constitutes a filtered contraction of coaugmented differential graded coalgebras. Here $\mathcal{S}^{\mathrm{c}} \nabla$ and $\mathcal{S}^{\mathrm{c}} \pi$ are morphisms of differential graded coalgebras but, beware, even though $\mathrm{T}^{\mathrm{c}} h$ is compatible with the coalgebra structure in the sense that it is a homotopy of morphisms of differential graded coalgebras, $\mathcal{S}^{\mathrm{c}} h$ no longer has such a compatibility property in a naive fashion. Indeed, for cocommutative coalgebras, the notion of homotopy is a subtle concept, cf. [45].

Definition 2.6. Given a chain complex $\mathfrak{g}$, an sh-Lie structure or $L_{\infty}$-structure on $\mathfrak{g}$ is a coalgebra perturbation $\partial$ of the differential $d$ on the coaugmented differential graded symmetric coalgebra $\mathcal{S}^{\mathrm{c}}[s \mathfrak{g}]$ on $s \mathfrak{g}$, that is, an operator $\partial$ of degree -1 which is compatible with the coalgebra structure, lowers filtration by 1 , and satisfies

$$
(d+\partial)^{2}=0
$$


or, equivalently,

$$
[d, \partial]+\partial \partial=0
$$

and, furthermore,

$$
\partial \eta=0
$$

so that the sum $d+\partial$ endows $\mathcal{S}^{\mathrm{c}}[s \mathfrak{g}]$ with a new coaugmented differential graded coalgebra structure.

In contrast to the case of a strict $\mathrm{dg}$ Lie algebra, here we may have non-zero terms $\mathcal{S}_{k}^{\mathrm{c}}[s \mathfrak{g}] \rightarrow s \mathfrak{g}$ for $k \geq 2$. For example, the Jacobi identity may hold only modulo an exact term provided by a morphism of the kind $\mathcal{S}_{3}^{\mathrm{c}}[s \mathfrak{g}] \rightarrow s \mathfrak{g}$.

Given an sh-Lie structure $\partial$ on $\mathfrak{g}$, we write $\mathcal{S}_{\partial}^{\mathrm{c}}[s \mathfrak{g}]$ for the new coaugmented differential graded coalgebra. We have explained in Proposition 1.3 how a graded Lie bracket $[\cdot, \cdot]$ on $\mathfrak{g}$ yields such a coalgebra perturbation $\partial$; the resulting chain complex $\mathcal{S}_{\partial}^{\mathrm{c}}[s \mathfrak{g}]$ then boils down to the generalized Koszul complex denoted there by $\mathcal{S}_{[\cdot, \cdot]}^{\mathrm{c}}[s \mathfrak{g}]$ or just $\mathcal{C}[\mathfrak{g}]$.

Given two sh-Lie algebras $\left(\mathfrak{g}_{1}, \partial_{1}\right)$ and $\left(\mathfrak{g}_{2}, \partial_{2}\right)$, an sh-morphism or sh-Lie map from $\left(\mathfrak{g}_{1}, \partial_{1}\right)$ to $\left(\mathfrak{g}_{2}, \partial_{2}\right)$ is a morphism $\mathcal{S}_{\partial_{1}}^{\mathrm{c}}\left[s \mathfrak{g}_{1}\right] \rightarrow \mathcal{S}_{\partial_{2}}^{\mathrm{c}}\left[s \mathfrak{g}_{2}\right]$ of differential graded coalgebras.

Theorem 2.7. Given a differential graded Lie algebra $\mathfrak{g}$ and a contraction of chain complexes of the kind (2.2), the corresponding coalgebra perturbation of the differential on $\mathcal{S}^{\mathrm{c}}[s \mathfrak{g}]$ being written $\partial$, the data determine

(i) a differential $\mathcal{D}$ on $\mathcal{S}^{\mathrm{c}}[s \mathrm{H}(\mathfrak{g})]$ turning the latter into a coaugmented differential graded coalgebra (i. e. $\mathcal{D}$ is a coalgebra perturbation of the zero differential) and hence endowing $\mathrm{H}(\mathfrak{g})$ with an sh-Lie algebra structure and

(ii) a Lie algebra twisting cochain $\tau: \mathcal{S}_{\mathcal{D}}^{\mathrm{c}}[s \mathrm{H}(\mathfrak{g})] \rightarrow \mathfrak{g}$ whose adjoint $\bar{\tau}$, written $\left(\mathcal{S}^{\mathrm{c}} \nabla\right)_{\partial}: \mathcal{S}_{\mathcal{D}}^{\mathrm{c}}[s \mathrm{H}(\mathfrak{g})] \rightarrow \mathcal{C}[\mathfrak{g}]$, induces an isomorphism on homology.

Furthermore, $\left(\mathcal{S}^{\mathrm{c}} \nabla\right)_{\text {д }}$ admits an extension to a new contraction

$$
\left(\mathcal{S}_{\mathcal{D}}^{\mathrm{c}}[s \mathrm{H}(\mathfrak{g})] \underset{\left(\mathcal{S}^{\mathrm{c}} \nabla\right)_{\partial}}{\stackrel{\left(\mathcal{S}^{\mathrm{c}} \pi\right)_{\partial}}{\leftrightarrows}} \mathcal{S}_{\partial}^{\mathrm{c}}[s \mathfrak{g}],\left(\mathcal{S}^{\mathrm{c}} h\right)_{\partial}\right)
$$

of filtered chain complexes (not nececessarily of coalgebras).

For intelligibility we point out that, according to the convention introduced before, in the statement of the theorem, the perturbed differential graded coalgebra which corresponds to the asserted sh-Lie algebra structure on $\mathrm{H}(\mathfrak{g})$ is written $\mathcal{S}_{\mathcal{D}}^{\mathrm{c}}[s \mathrm{H}(\mathfrak{g})]$. Further, the morphisms $\left(\mathcal{S}^{\mathrm{c}} \nabla\right)_{\partial}$ and $\left(\mathcal{S}^{\mathrm{c}} \pi\right)_{\partial}$ coming into play in the new contraction (2.7.1) are sh-Lie maps between $(\mathrm{H}(\mathfrak{g}), \mathcal{D})$ and $(\mathfrak{g}, \partial)$. They are even sh-equivalences (quasi-isomorphisms) in the sense that they induce isomorphisms on homology. It is worthwhile pointing out that the induced bracket on $\mathrm{H}(\mathfrak{g})$ is a strict graded Lie bracket but, in general, the differential $\mathcal{D}$ involves meaningful terms of higher order.

Sketch of the proof of Theorem 2.7. For $b \geq 1$, write $\mathcal{S}_{b}^{c}$ for the homogeneous degree $b$ component of $\mathcal{S}^{\mathrm{c}}[s \mathrm{H}(\mathfrak{g})]$. The operator $\mathcal{D}$ and twisting cochain $\tau$ are obtained as 
infinite series by the following recursive procedure where $b \geq 2$ :

$$
\begin{aligned}
\tau & =\tau^{1}+\tau^{2}+\ldots, \quad \tau^{1}=\nabla \tau_{\mathrm{H}(\mathfrak{g})}, \quad \tau^{j}: \mathcal{S}_{j}^{\mathrm{c}} \rightarrow \mathfrak{g}, \quad j \geq 1, \\
\tau^{b} & =\frac{1}{2} h\left(\left[\tau^{1}, \tau^{b-1}\right]+\cdots+\left[\tau^{b-1}, \tau^{1}\right]\right) \\
\mathcal{D} & =\mathcal{D}^{1}+\mathcal{D}^{2}+\ldots
\end{aligned}
$$

where $\mathcal{D}^{b-1}$ is the coderivation of $\mathcal{S}^{\mathrm{c}}[s \mathrm{H}(\mathfrak{g})]$ determined by

$$
\tau_{\mathrm{H}(\mathfrak{g})} \mathcal{D}^{b-1}=\frac{1}{2} \pi\left(\left[\tau^{1}, \tau^{b-1}\right]+\cdots+\left[\tau^{b-1}, \tau^{1}\right]\right): \mathcal{S}_{b}^{\mathrm{c}} \rightarrow \mathrm{H}(\mathfrak{g}) .
$$

The sums (2.7.2) and (2.7.3) are infinite but, applied to a specific element which, in view of the assumptions, necessarily lies in some finite filtration degree subspace, only finitely many terms will be non-zero, whence the convergence is naive. For example, cf. (2.7.3), for $n \geq 1$, the operator $\mathcal{D}^{n}$ vanishes on the constituent $F_{n}$ of the coaugmentation filtration

$$
R=F_{0} \subseteq F_{1} \subseteq F_{2} \subseteq \ldots
$$

of $\mathcal{S}^{\mathrm{c}}[s \mathrm{H}(\mathfrak{g})]$. Since the coaugmentation filtration is cocomplete, if the infinite sum (2.7.3) for $\mathcal{D}$ is applied to a particular element, only finitely many terms are non-zero. The summand $\mathcal{D}^{1}$ is the ordinary Cartan-Chevalley-Eilenberg differential for the classifying coalgebra $\mathcal{C}[\mathrm{H}(\mathfrak{g})]$ of the graded Lie algebra $\mathrm{H}(\mathfrak{g})$. It may be shown that $\mathcal{D}$ is indeed a coalgebra differential and that $\tau$ is a twisting cochain; the details will be given elsewhere. A construction which is formally similar but yet substantially different may be found in Proposition 2.1 of [15].

A spectral sequence comparison argument shows that the adjoint $\bar{\tau}=\left(\mathcal{S}^{c} \nabla\right)_{\partial}$ of $\tau$ induces an isomorphism on homology. Hence $\left(\mathcal{S}^{c} \nabla\right)_{\partial}$ admits an extension to a contraction of chain complexes of the kind (2.7.1).

\subsection{ReFinements.}

Addendum 2.8.1. Under the circumstances of Theorem 2.7,

$$
\tau: \mathcal{S}_{\mathcal{D}}^{\mathrm{c}}[s \mathrm{H}(\mathfrak{g})] \rightarrow \mathfrak{g},
$$

viewed as an element of degree -1 of the differential graded Lie algebra $\operatorname{Hom}\left(\mathcal{S}_{\mathcal{D}}^{\mathrm{c}}[s \mathrm{H}(\mathfrak{g})], \mathfrak{g}\right)$, satisfies the master equation (1.4.2).

Here $\operatorname{Hom}\left(\mathcal{S}_{\mathcal{D}}^{\mathrm{c}}[s \mathrm{H}(\mathfrak{g})], \mathfrak{g}\right)$ is endowed with the graded cup bracket (1.5.1) induced by the graded coalgebra structure on $\mathcal{S}_{\mathcal{D}}^{\mathrm{c}}[s \mathrm{H}(\mathfrak{g})]$ and the graded bracket on $\mathfrak{g}$. The twisting cochain (2.8.2) is our most general solution of the master equation; the other (more special) solutions of the master equation derive from it.

Addendum 2.8.3. Under the circumstances of Theorem 2.7, suppose in addition that there is a differential $\widetilde{\mathcal{D}}$ on $\mathcal{S}^{\mathrm{c}}[s \mathrm{H}(\mathfrak{g})]$ turning the latter into a coaugmented differential graded coalgebra in such a way that $\left(\mathcal{S}^{\mathrm{c}} \pi\right) \partial=\widetilde{\mathcal{D}}\left(\mathcal{S}^{\mathrm{c}} \pi\right)$. Then $\mathcal{D}=\widetilde{\mathcal{D}}$ and 
$\left(\mathcal{S}^{\mathrm{c}} \pi\right)_{\text {a }}$ may be taken to be $\mathcal{S}^{\mathrm{c}} \pi$. In particular, when $\left(\mathcal{S}^{\mathrm{c}} \pi\right) \partial$ is zero, the differential $\mathcal{D}$ on $\mathcal{S}^{\mathrm{c}}[s \mathrm{H}(\mathfrak{g})]$ is necessarily zero, that is, the new contraction (2.7.1) has the form

$$
\left(\mathcal{S}^{\mathrm{c}}[s \mathrm{H}(\mathfrak{g})] \underset{\left(\mathcal{S}^{\mathrm{c}} \nabla\right)_{\partial}}{\leftrightarrows} \mathcal{S}_{\partial}^{\mathrm{c}}[s \mathfrak{g}],\left(\mathcal{S}^{\mathrm{c}} h\right)_{\partial}\right)
$$

For example, this happens to be the case when the composite $\mathfrak{g} \otimes \mathfrak{g} \stackrel{[\cdot, \cdot]}{\longrightarrow} \mathfrak{g} \stackrel{\pi}{\longrightarrow} \mathrm{H}(\mathfrak{g})$ is zero.

In fact, this follows at once from the descriptions (2.7.3) and (2.7.2) for $\mathcal{D}$ and $\tau$ : Since $\left(\mathcal{S}^{\mathrm{c}} \pi\right) \partial=\widetilde{\mathcal{D}}\left(\mathcal{S}^{\mathrm{c}} \pi\right)$, only the quadratic part $\widetilde{\mathcal{D}}^{1}: \mathcal{S}_{2}^{\mathrm{c}} \rightarrow s \mathrm{H}(\mathfrak{g})$ of $\widetilde{\mathcal{D}}$ is non-zero and this quadratic part necessarily coincides with the quadratic part $\mathcal{D}^{1}$ of $\mathcal{D}$. Furthermore, since the constituent $\pi$ of the contraction $(2.2)$ is necessarily a morphism of differential graded Lie algebras, $\mathcal{D}$ has no higher terms as well. Indeed, since $\pi h=0$, for $b \geq 2, \pi \tau^{b}=0$ whence $\pi\left[\tau^{j}, \tau^{k}\right]=\left[\pi \tau^{j}, \pi \tau^{k}\right](j, k \geq 1)$ is non-zero only for $j=k=1$. Hence $\mathcal{D}^{b}$ is zero for $b \geq 2$.

Likewise, we have the following.

Addendum 2.8.5. Under the circumstances of Theorem 2.7, suppose in addition that there is a differential $\widetilde{\mathcal{D}}$ on $\mathcal{S}^{\mathrm{c}}[s \mathrm{H}(\mathfrak{g})]$ turning the latter into a coaugmented differential graded coalgebra in such a way that $\partial\left(\mathcal{S}^{c} \nabla\right)=\left(\mathcal{S}^{c} \nabla\right) \widetilde{\mathcal{D}}$. Then $\mathcal{D}=\widetilde{\mathcal{D}}$ and $\left(\mathcal{S}^{\mathrm{c}} \nabla\right)_{\partial}=\mathcal{S}^{\mathrm{c}} \nabla$. In particular, when $\partial\left(\mathcal{S}^{\mathrm{c}} \nabla\right)$ is zero, the differential $\mathcal{D}$ on $\mathcal{S}^{\mathrm{c}}[s \mathrm{H}(\mathfrak{g})]$ is necessarily zero, that is, the new contraction (2.7.1) has the form

$$
\left(\mathcal{S}^{\mathrm{c}}[s \mathrm{H}(\mathfrak{g})] \underset{\mathcal{S}^{\mathrm{c}} \nabla}{\stackrel{\left(\mathcal{S}^{\mathrm{c}} \pi\right)_{\partial}}{\rightleftarrows}} \mathcal{S}_{\partial}^{\mathrm{c}}[s \mathfrak{g}],\left(\mathcal{S}^{\mathrm{c}} h\right)_{\partial}\right) .
$$

For example, this happens to be the case when the composite $\mathrm{Hg} \otimes \mathrm{Hg} \stackrel{\nabla \otimes \nabla}{\longrightarrow} \mathfrak{g} \otimes \mathfrak{g} \stackrel{[\cdot, \cdot]}{\longrightarrow} \mathfrak{g}$ is zero.

In the case of (2.8.3), $\pi: \mathfrak{g} \rightarrow \mathrm{H}(\mathfrak{g})$ is in fact a strict graded Lie morphism and so is, likewise, $\nabla: \mathrm{H}(\mathfrak{g}) \rightarrow \mathfrak{g}$ in the case of (2.8.5). We note that observations similar to these addenda may be found in (2.4.1) and (2.4.2) of [22].

Under the circumstances of Theorem 2.7 where $\mathfrak{g}$ is a true differential graded Lie algebra, the perturbation $\mathcal{D}$ still endows $\mathrm{H}(\mathfrak{g})$ with an sh-Lie algebra structure which is in general non-trivial, and this will be so even if the induced Lie bracket on $\mathrm{H}(\mathfrak{g})$ is zero but not if an additional (technical) condition (which arises from abstraction from the statement of the $\partial \bar{\partial}$-Lemma for Kähler manifolds, see what is said below) is satisfied, according to Addendum 2.8.3. This latter is the situation for Barannikov-Kontsevich [1] and Manin [38] (III.10), [39] (Section 6). They have stronger conditions in the main applications, for which we spell out the following.

Theorem 2.9. Given a differential graded Lie algebra $\mathfrak{g}$, a differential graded Lie subalgebra $\mathfrak{m}$ of $\mathfrak{g}$, and a contraction

$$
(\mathrm{H}(\mathfrak{g}) \stackrel{\pi}{\stackrel{\stackrel{\pi}{\leftrightarrows}}{\leftrightarrows}} \mathfrak{m}, h)
$$

of chain complexes so that the composite $\mathfrak{m} \otimes \mathfrak{m} \stackrel{[\cdot, \cdot]}{\longrightarrow} \mathfrak{m} \stackrel{\pi}{\longrightarrow} \mathrm{H}(\mathfrak{g})$ is zero, the induced bracket on $\mathrm{H}(\mathfrak{g})$ is zero, that is, as a graded Lie algebra, $\mathrm{H}(\mathfrak{g})$ is abelian, and the 
data determine a solution $\tau \in \operatorname{Hom}\left(\mathcal{S}^{\mathrm{c}}[s \mathrm{H}(\mathfrak{g})], \mathfrak{g}\right)$ of the master equation

$$
d \tau=\frac{1}{2}[\tau, \tau]
$$

in such a way that the following hold:

(i) The composite $\pi \tau$ coincides with the universal twisting cochain $\mathcal{S}^{\mathrm{c}}[s \mathrm{H}(\mathfrak{g})] \rightarrow \mathrm{H}(\mathfrak{g})$ for the abelian graded Lie algebra $\mathrm{H}(\mathfrak{g})$;

(ii) the values of $\tau$ lie in $\mathfrak{m}$.

Notice that (2.9.2) is somewhat simpler than the general master equation (1.4.2) since there is no non-zero differential on $\mathcal{S}^{\mathrm{c}}[s \mathrm{H}(\mathfrak{g})]$.

Proof. Under these circumstances, the homology of $\mathfrak{m}$ is necessarily identified with $\mathrm{H}(\mathfrak{g})$ under (2.9.1). In view of Addendum 2.8.3, the corresponding contraction (2.7.1) has the form

$$
\left(\mathcal{S}^{\mathrm{c}}[s \mathrm{H}(\mathfrak{g})] \underset{\left(\mathcal{S}^{\mathrm{c}} \nabla\right)_{\partial}}{\leftrightarrows} \mathcal{S}_{\partial}^{\mathrm{c}}[s \mathfrak{m}],\left(\mathcal{S}^{\mathrm{c}} h\right)_{\partial}\right)
$$

Notice that the differential on $\mathcal{S}^{\mathrm{c}}[s \mathrm{H}(\mathfrak{g})]$ is zero. Consider the corresponding Lie algebra twisting cochain (2.8.2) (where the role of $\mathfrak{g}$ in (2.8.2) is now played by $\mathfrak{m}$ ) which we write as

$$
\widetilde{\tau}=\tau_{\mathfrak{m}} \circ\left(\mathcal{S}^{\mathrm{c}} \nabla\right)_{\partial}: \mathcal{S}^{\mathrm{c}}[s \mathrm{H}(\mathfrak{g})] \rightarrow \mathfrak{m}
$$

and denote by $\tau$ its composite with the inclusion $\mathfrak{m} \subseteq \mathfrak{g}$. Since this inclusion is a morphism of differential graded Lie algebras, $\tau$ has the asserted properties.

\section{Differential Gerstenhaber and Batalin-Vilkovisky algebras}

Recall that a Gerstenhaber algebra is a graded commutative $R$-algebra $\mathcal{A}$ together with a graded Lie bracket from $\mathcal{A} \otimes_{R} \mathcal{A}$ to $\mathcal{A}$ of degree -1 (in the sense that, if $\mathcal{A}$ is regraded down by one, [., $\cdot]$ is an ordinary graded Lie bracket) such that, for each homogeneous element $a$ of $\mathcal{A}$, the operation $[a, \cdot]$ is a derivation of $\mathcal{A}$ of degree $|a|-1$ where $|a|$ refers to the degree of $a$; see [12] where these objects are called G-algebras, or $[34,37,48]$; for a Gerstenhaber algebra $\mathcal{A}$, the bracket from $\mathcal{A} \otimes_{R} \mathcal{A}$ to $\mathcal{A}$ will henceforth be referred to as its Gerstenhaber bracket.

Definition 3.1. A differential Gerstenhaber algebra $(\mathcal{A},[\cdot, \cdot], d)$ consists of a Gerstenhaber algebra $(\mathcal{A},[\cdot, \cdot])$ and a differential $d$ of degree +1 on $\mathcal{A}$ which is a derivation for the multiplication of $\mathcal{A} ;(\mathcal{A},[\cdot, \cdot], d)$ will be said to be a strict differential Gerstenhaber algebra provided the differential $d$ is a derivation for the Gerstenhaber bracket $[\cdot, \cdot]$ as well, that is,

$$
d[x, y]=[d x, y]-(-1)^{|x|}[x, d y], \quad x, y \in \mathcal{A}
$$

Let $(\mathcal{A},[\cdot, \cdot], d)$ be a strict differential Gerstenhaber algebra. We denote the corresponding ordinary differential graded Lie algebra, with differential spelled out as a homology differential, by $\mathfrak{g}^{\mathcal{A}}$ or, more simply, by $\mathfrak{g}$, when there is no risk of confusion. Thus, $\mathfrak{g}_{-*}=\mathcal{A}^{*+1}$, that is to say,

$$
\mathfrak{g}_{1}=\mathcal{A}^{0}, \quad \mathfrak{g}_{0}=\mathcal{A}^{1}, \quad \mathfrak{g}_{-1}=\mathcal{A}^{2}, \quad \ldots, \quad \mathfrak{g}_{-n}=\mathcal{A}^{n+1}
$$


so that the graded bracket $[\cdot, \cdot]$ and differential $d$ on $\mathfrak{g}$ are of the ordinary kind, i. e. of the form

$$
[\cdot, \cdot]: \mathfrak{g}_{j} \otimes \mathfrak{g}_{k} \rightarrow \mathfrak{g}_{j+k}, \quad d: \mathfrak{g}_{j} \rightarrow \mathfrak{g}_{j-1} .
$$

Notice that, when we write $\mathfrak{a}_{-*}=\mathcal{A}^{*}$, so that $\mathfrak{a}_{0}=\mathcal{A}^{0}, \mathfrak{a}_{-1}=\mathcal{A}^{1}$, etc., we have $\mathfrak{g}=s \mathfrak{a}$ and $\mathfrak{a}=s^{-1} \mathfrak{g}$. The notation $\mathfrak{a}$ is just used to rewrite the strict differential Gerstenhaber algebra $\mathcal{A}$ in homology degrees.

Consider a contraction of the kind (2.2). Application of Theorem 2.7, the operator $\partial$ on $\mathcal{S}_{\partial}^{\mathrm{c}}[s \mathfrak{g}]$ which corresponds to the Lie bracket on $\mathfrak{g}$ being regarded as the perturbation to be transferred (to $\mathcal{S}^{\mathrm{c}}[s \mathrm{H}(\mathfrak{g})]$ ), yields a new contraction

$$
\left(\mathcal{S}_{\mathcal{D}}^{\mathrm{c}}[s \mathrm{H}(\mathfrak{g})] \underset{\left(\mathcal{S}^{\mathrm{c}} \nabla\right)_{\partial}}{\stackrel{\left(\mathcal{S}^{\mathrm{c}} \pi\right)_{\partial}}{\leftrightarrows}} \mathcal{S}_{\partial}^{\mathrm{c}}[s \mathfrak{g}],\left(\mathcal{S}^{\mathrm{c}} h\right)_{\partial}\right)
$$

of filtered differential graded coalgebras. The corresponding Lie algebra twisting cochain denoted in (2.8.2) by $\tau$, now written out as having $\mathfrak{a}$ as its target rather than $\mathfrak{g}$, appears as an element $\tau \in \operatorname{Hom}\left(\mathcal{S}_{\mathcal{D}}^{\mathrm{c}}[s \mathrm{H}(\mathfrak{g})], \mathfrak{a}\right)$ of degree -2 satisfying the master equation

$$
D \tau=\frac{1}{2}[\tau, \tau] .
$$

Here $D$ is the Hom-differential, and $[\cdot, \cdot]$ refers to the graded cup bracket (1.5.1) which is induced by the graded coalgebra structure on $\mathcal{S}^{\mathrm{c}}[s \mathrm{H}(\mathfrak{g})]$ and the graded Lie structure on $\mathfrak{g}$ which, in turn, is the graded Gerstenhaber bracket on $\mathfrak{a}$.

For consistency with what is in the literature, we now rewrite $\operatorname{Hom}\left(\mathcal{S}_{\mathcal{D}}^{\mathrm{c}}[s \mathrm{H}(\mathfrak{g})], \mathfrak{a}\right)$ and the element $\tau$ thereof in terms of cohomology degrees: Let $\mathcal{G}^{*}=\mathfrak{g}_{*}$; in this description, $\mathcal{A}=S \mathcal{G}$, where $S$ refers to the suspension operator in cohomology degrees, so that, for every integer $j$,

$$
(\mathcal{A})^{j}=(S \mathcal{G})^{j}=\mathcal{G}^{j+1}, \quad\left(S^{-1} \mathrm{H}^{*}(\mathcal{G})\right)^{j}=\mathrm{H}^{j+1}(\mathcal{G}) .
$$

The twisting cochain $\tau$ now appears as an element

$$
\tau \in \mathcal{B}=\operatorname{Hom}\left(\mathcal{S}_{\mathcal{D}}^{\mathrm{c}}\left[S^{-1} \mathrm{H}(\mathcal{G})\right], \mathcal{A}\right)
$$

of degree +2 satisfying the master equation.

Definition 3.5. For a Gerstenhaber algebra $\mathcal{A}$ over $R$, with bracket operation written $[\cdot, \cdot]$, an $R$-linear operator $\Delta$ on $\mathcal{A}$ of degree -1 is said to generate the Gerstenhaber bracket provided, for every homogeneous $a, b \in \mathcal{A}$,

$$
[a, b]=(-1)^{|a|}\left(\Delta(a b)-(\Delta a) b-(-1)^{|a|} a(\Delta b)\right) ;
$$

the operator $\Delta$ is then called a generator. A generator $\Delta$ is said to be exact provided $\Delta \Delta$ is zero, that is, $\Delta$ is a differential. A Gerstenhaber algebra $\mathcal{A}$ together with a generator $\Delta$ will be called a weak Batalin-Vilkovisky algebra (or weak $\mathrm{BV}$-algebra); when the generator is exact, $(\mathcal{A}, \Delta)$ is (more simply) called a Batalin-Vilkovisky algebra (or BV-algebra). The notation $\Delta$ for a generator of a Batalin-Vilkovisky algebra has become standard in the literature, and we stick to it. 
There is no conflict with the earlier notation $\Delta$ for the diagonal map of a coalgebra since diagonal maps will not appear explicitly any more.

It is clear that a generator determines the Gerstenhaber bracket. An observation due to Koszul [35] (p. 261) says that, for any Batalin-Vilkovisky algebra $(\mathcal{A},[\cdot, \cdot], \Delta)$, the operator $\Delta$ (which is exact by assumption) behaves as a derivation for the Gerstenhaber bracket $[\cdot, \cdot]$, that is,

$$
\Delta[x, y]=[\Delta x, y]-(-1)^{|x|}[x, \Delta y], \quad x, y \in \mathcal{A} .
$$

A generator $\Delta$, even if exact, behaves as a derivation for the multiplication of $\mathcal{A}$ only if the Gerstenhaber bracket is zero.

Definition 3.6. Let $(\mathcal{A}, \Delta)$ be a weak Batalin-Vilkovisky algebra, write $[\cdot, \cdot]$ for the Gerstenhaber bracket generated by $\Delta$, and let $d$ be a differential of degree 1 which endows $(\mathcal{A},[\cdot, \cdot])$ with a differential Gerstenhaber algebra structure. The triple $(\mathcal{A}, \Delta, d)$ is called a weak differential Batalin-Vilkovisky algebra provided the graded commutator $[d, \Delta]=d \Delta+\Delta d$ on $\mathcal{A}$ (which is a degree zero operator) is zero. In particular, a weak differential Batalin-Vilkovisky algebra $(\mathcal{A}, \Delta, d)$ which has $\Delta$ exact is called a differential Batalin-Vilkovisky algebra.

Proposition 3.7. For any weak differential Batalin-Vilkovisky algebra $(\mathcal{A}, \Delta, d)$, the differential $d$ behaves as a derivation for the Gerstenhaber bracket $[\cdot, \cdot]$ on $\mathcal{A}$ generated by $\Delta$, that is to say,

$$
d[x, y]=[d x, y]-(-1)^{|x|}[x, d y], \quad x, y \in \mathcal{A} .
$$

In other words, $(\mathcal{A},[\cdot, \cdot], d)$ is a differential Gerstenhaber algebra.

Proof. This is well known; the reader is invited to concoct a proof himself.

Notice that, under the circumstances of (3.7), $\Delta$ need not behave as a derivation for the Gerstenhaber bracket unless $\Delta$ is exact.

Barannikov-Kontsevich [1] and Manin [38] (III.10.1.1), [39] (6.1.1), are concerned not just with a Batalin-Vilkovisky algebra $\mathcal{A}$ for which $\mathrm{H}(\mathfrak{g})$ is abelian, but moreover with one which satisfies the formalization of the ordinary formality Lemma for Kähler manifolds. This leads to a situation of exactly the kind isolated in Theorem 2.9. We now explain this in our framework.

We will say that a differential Batalin-Vilkovisky algebra $(\mathcal{A}, \Delta, d)$ satisfies the statement of the Kählerian formality lemma provided the maps

$$
\left(\operatorname{ker}(\Delta),\left.d\right|_{\operatorname{ker}(\Delta)}\right) \stackrel{\subseteq}{\longrightarrow}(\mathcal{A}, d), \quad\left(\operatorname{ker}(\Delta),\left.d\right|_{\operatorname{ker}(\Delta)}\right) \stackrel{\operatorname{proj}}{\longrightarrow} \mathrm{H}(\mathcal{A}, \Delta)
$$

are isomorphisms on homology where $\mathrm{H}(\mathcal{A}, \Delta)$ is endowed with the zero differential.

What we mean by the Kählerian formality lemma is more usually referred to as

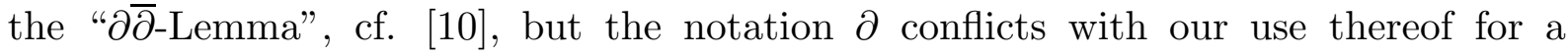
perturbation to which we will stick.

Theorem 3.8. Let $(\mathcal{A}, \Delta, d)$ be a differential Batalin-Vilkovisky algebra satisfying the statement of the Kählerian formality lemma, let $\mathfrak{g}$ be the differential graded Lie algebra related with the underlying differential $\operatorname{Gerstenhaber}(\mathcal{A},[\cdot, \cdot], d)$ as in $(3.2)$ above, and extend the projection proj to a contraction

$$
(\mathrm{H}(\mathfrak{g}) \stackrel{\pi}{\stackrel{\pi}{\leftrightarrows}} \mathfrak{m}, h)
$$


of chain complexes, where $\mathfrak{m}=\left(\operatorname{ker}(\Delta),\left.d\right|_{\operatorname{ker}(\Delta)}\right)$ and $\pi=$ proj. Then, as a graded Lie algebra, $\mathrm{H}(\mathfrak{g})$ is abelian, and the data determine a solution $\tau \in \operatorname{Hom}\left(\mathcal{S}^{\mathrm{c}}[s \mathrm{H}(\mathfrak{g})], \mathfrak{g}\right)$ of the master equation $d \tau=\frac{1}{2}[\tau, \tau]$ in such a way that the following hold:

(i) The values of $\tau$ lie in $\mathfrak{m}$, that is, the composite $\Delta \circ \tau: \mathcal{S}^{\mathrm{c}}[s \mathrm{H}(\mathfrak{g})] \rightarrow \mathfrak{g}$ is zero;

(ii) the composite $\pi \tau$ coincides with the universal twisting cochain $\mathcal{S}^{\mathrm{c}}[s \mathrm{H}(\mathfrak{g})] \rightarrow \mathrm{H}(\mathfrak{g})$ for the abelian graded Lie algebra $\mathrm{H}(\mathfrak{g})$; whence

(iii) for $k \geq 2$, the values of the component $\tau_{k}$ on $\mathcal{S}_{k}^{\mathrm{c}}[s \mathrm{H}(\mathfrak{g})]$ of $\tau$ lie in $\mathrm{im} \Delta$.

Proof. This in an immediate consequence of Theorem 2.9 except statement (iii) which, in view of (i) and (ii), follows from the exactness of the sequence

$$
0 \rightarrow \operatorname{im} \Delta \rightarrow \operatorname{ker} \Delta \rightarrow \mathrm{Hg} \rightarrow 0
$$

and the fact that, for $k \geq 2$, the component on $\mathcal{S}_{k}^{\mathrm{c}}[s \mathrm{H}(\mathfrak{g})]$ of the universal twisting cochain for $\mathrm{H}(\mathfrak{g})$ is zero, cf. (1.5.5).

Under the circumstances of (3.8) suppose that, in degree $0, \mathcal{A}$ consists of a single copy of the ground ring $R$ necessarily generated by the unit 1 of $\mathcal{A}$ and that $\Delta(1)$ is zero. Then 1 generates a central copy of $R$ in $\mathfrak{g}$, and we may write $\mathfrak{g}$ as a direct sum $R \oplus \widetilde{\mathfrak{g}}$ of differential graded Lie algebras where $\widetilde{\mathfrak{g}}$ is the uniquely determined complement of $R$ in $\mathfrak{g}$ and $\widetilde{\mathfrak{g}}$ is itself a differential graded Lie algebra.

Addendum 3.8.2. Under these circumstances, if the class [1] in homology is nonzero, the requisite contraction (3.8.1) may be chosen in such a way that, for $k \geq 2$, the values of the $\tau_{k}$ lie in $\tilde{\mathfrak{g}}$.

Proof. The differential graded Lie algebra $\mathfrak{m}$ decomposes accordingly as a direct sum $R \oplus \widetilde{\mathfrak{m}}$ of differential graded Lie algebras and so does the homology of $\mathfrak{g}$, i. e. it decomposes as $\mathrm{H}(\mathfrak{g})=R \oplus \mathrm{H}(\mathfrak{g})$. The projection proj from $\operatorname{ker} \Delta$ to $\mathrm{H}(\mathcal{A}, \Delta)$ may thus be extended to a contraction of chain complexes $(R \oplus \mathrm{H}(\widetilde{\mathfrak{g}}) \stackrel{\stackrel{\pi}{\nabla}}{\leftrightarrows} \oplus \widetilde{\mathfrak{m}}, h)$ in such a way that the morphisms $\nabla, \pi$ and $h$ decompose accordingly; in particular, we have a contraction $(\mathrm{H}(\tilde{\mathfrak{g}}) \underset{\widetilde{\nabla}}{\stackrel{\widetilde{\pi}}{\leftrightarrows}} \widetilde{\mathfrak{m}}, \widetilde{h})$. Let $\widetilde{\tau}: \mathcal{S}^{\mathrm{c}}[s \mathrm{H}(\widetilde{\mathfrak{g}})] \rightarrow \widetilde{\mathfrak{g}}$ be the twisting cochain resulting from applying Theorem 2.9 to this contraction and define

$$
\tau: \mathcal{S}^{\mathrm{c}}[s \mathrm{H}(\mathfrak{g})]=\mathcal{S}^{\mathrm{c}}[s \mathrm{H}(\widetilde{\mathfrak{g}})] \otimes \mathcal{S}^{\mathrm{c}}[s R] \rightarrow R \oplus \mathrm{H}(\widetilde{\mathfrak{g}})=\mathfrak{g}
$$

by $\tau=\varepsilon \otimes \widetilde{\tau}+\tau_{0} \otimes \varepsilon$ where $\tau_{0}$ refers to the universal twisting cochain $\tau_{0}: \mathcal{S}^{\mathrm{c}}[s R] \rightarrow R$ for the abelian Lie algebra $R$ and $\varepsilon$ to the corresponding counits. The twisting cochain $\tau$ has the desired properties.

Apart from the context, Theorem 3.8 and the Addendum 3.8.2 contain Lemma 6.1 in [1]. More precisely, condition (6.1 (1)) in [1], referred to there as the universality condition, translates to the fact that a contraction is a very precise way of spelling out a homology isomorphism. Furthermore, the condition (6.1 (2)) in [1], referred to by the wording flat coordinates, is implied by (i) and (iii) in Theorem 3.8. The statement of the Addendum amounts to Barannikov and Kontsevich's "flat identity" property. A statement of the kind (ii) in Theorem 3.8 is referred to by the wording normalized in Theorem III.9.2 of [38] and Theorem 4.2 of [39]. We note that 
a contraction may in fact carry additional information which could be physically relevant, e. g. in terms of higher order correlation functions.

\section{Deformation theory}

Barannikov and Kontsevich set up their construction in the context of deformation theory. In particular, they show that one very specific differential graded Lie algebra which arises from a Calabi-Yau manifold is formal and that its formal moduli space which, in a sense, is the formal space of equivalence classes of solutions of the relevant master equation, is affine with a very simple (coordinate) description. Our method applies much more generally. Given a differential graded Lie algebra $\mathfrak{g}$ over the reals or over any field $\mathbf{k}$ of characteristic zero, the construction of the solution $\tau$ given above as (2.8.2) can be carried out once the requisite contraction of the kind (2.2) has been chosen (over a field of characteristic zero, such a choice is always possible, as we remarked earlier) and still yields a formal solution of the master equation, but with a perturbed differential on $\mathcal{S}^{\mathrm{c}}[s \mathrm{Hg}]$, starting with the operator induced by the Lie bracket of $\mathrm{Hg}$. The moduli space interpretation is then available along the lines of that of Schlessinger-Stasheff [45] used for the moduli space of rational homotopy types, once a cohomology algebra is fixed in advance. One major difference is that the resulting "versal moduli" space need no longer be affine. We now explain this briefly.

Let $L$ be a differential graded Lie algebra over a field $\mathbf{k}$ of characteristic zero which, viewed as graded by cohomology degrees, is concentrated in nonnegative degrees. Thus we have homogeneous components $L^{0}, L^{1}, \ldots$, and the differential $d$ is an operator of the form $d: L^{j} \rightarrow L^{j+1}$. The construction in Theorem 2.7 then yields a coalgebra perturbation $\mathcal{D}$ on $\mathcal{S}^{\mathrm{c}}\left[S^{-1} \mathrm{H}(L)\right]$ turning the latter into a differential graded coalgebra, together with a Lie algebra twisting cochain

$$
\tau: \mathcal{S}_{\mathcal{D}}^{\mathrm{c}}\left[S^{-1} \mathrm{H}(L)\right] \rightarrow L .
$$

Under the circumstances explained in Theorem $3.8, \mathcal{D}$ is zero, and this reflects the formality of the corresponding differential graded Lie algebra. Pursuing the philosophy in [45] we now offer an interpretation for a non-zero $\mathcal{D}$.

Recall that a possible deformation theory interpretation proceeds as follows: Let $V_{L} \subseteq L^{1}$ be the space of "integrable elements", or "perturbations", or solutions $\gamma \in L^{1}$ of the master equation $d \gamma=\frac{1}{2}[\gamma, \gamma]$. Under suitable circumstances, $V_{L}$ is a quadratic variety (in the appropriate sense). When $\mathbf{k}$ is the field of real numbers $\mathbb{R}$ or that of complex numbers $\mathbb{C}$, we may consider the Lie group $\mathcal{L}=\exp \left(L^{0}\right)$ which corresponds to $L^{0}$ and, when we assume that the induced $\mathcal{L}$-action on $V_{L}$ is complete, we may consider the corresponding moduli space $M_{L}$, the space of $\mathcal{L}$-orbits in $V_{L}$. Taking an appropriate algebra $A\left(L^{1}\right)$ of functions on the affine space $L^{1}$ as coordinate ring, for example polynomial functions with respect to a basis, or formal power series, on $V_{L}$ we have the algebra $A\left(V_{L}\right)=A\left(L^{1}\right) / J$ of functions where $J$ is the ideal of functions in $A\left(L^{1}\right)$ which vanish on $V_{L}$; likewise, the algebra $A\left(M_{L}\right)=\left(A\left(L^{1}\right)\right)^{\mathcal{L}} / J^{\mathcal{L}}$ of $\mathcal{L}$-invariant functions in $A\left(L^{1}\right)$ modulo the ideal $J^{\mathcal{L}}$ of $\mathcal{L}$-invariant functions which vanish on $V_{L}$ appears as an algebra $A\left(M_{L}\right)$ of functions on the moduli space $M_{L}$, and we may view the algebra $A\left(M_{L}\right)$ as a coordinate ring for $M_{L}$. It is not a coordinate ring in the ordinary sense of algebraic geometry, though. 
Since $\mathcal{S}_{[\cdot, \cdot]}^{\mathrm{c}}\left[S^{-1}(L)\right]$ is bigraded, so is $\operatorname{Hom}\left(\mathcal{S}_{[\cdot, \cdot]}^{\mathrm{c}}\left[S^{-1}(L)\right], \mathbb{C}\right)$, and its (co)homology inherits a bigrading. Consider the bidegree $(0,0)$ cohomology

$$
\mathrm{H}^{0,0}\left(\operatorname{Hom}\left(\mathcal{S}_{[\cdot, \cdot]}^{\mathrm{c}}\left[S^{-1}(L)\right], \mathbb{C}\right)\right),
$$

that is, the subalgebra of the degree zero cohomology $\mathrm{H}^{0}\left(\operatorname{Hom}\left(\mathcal{S}_{[,, \cdot]}^{\mathrm{c}}\left[S^{-1}(L)\right], \mathbb{C}\right)\right)$ which is generated by cocycles of bidegree $(0,0)$. The essential observation is now that, in the formal sense, i. e. when we are working with formal power series, this algebra is closely related to the algebra $A\left(M_{L}\right)$ introduced before. (We leave open here the precise relationship between $A\left(M_{L}\right)$ and $\mathrm{H}^{0,0}\left(\operatorname{Hom}\left(\mathcal{S}_{[,, \cdot]}^{\mathrm{c}}\left[S^{-1}(L)\right], \mathbb{C}\right)\right)$.)

Over a general field $\mathbf{k}$ (of characteristic zero), we now change gears and view the bidegree zero cohomology algebra $\mathrm{H}^{0,0}\left(\operatorname{Hom}\left(\mathcal{S}_{[\cdot, \cdot]}^{\mathrm{c}}\left[S^{-1}(L)\right], \mathbf{k}\right)\right.$ ) of the (differential graded) k-valued Chevalley-Eilenberg algebra of $L$ as the coordinate ring $A\left(M_{L}\right)$ of the moduli space $M_{L}$, thereby defining this moduli space by its coordinate ring. Theorem 7.1 of [45] then entails the following interpretation: By construction, the morphism

$$
\left(\mathcal{S}^{\mathrm{c}} \nabla\right)_{\partial}: \mathcal{S}_{\mathcal{D}}^{\mathrm{c}}\left[S^{-1}(\mathrm{H}(L))\right] \rightarrow \mathcal{S}_{[\cdot, \cdot]}^{\mathrm{c}}\left[S^{-1}(L)\right]
$$

is compatible with the bigradings and a quasi isomorphism whence $\left(\mathcal{S}^{\mathrm{c}} \nabla\right)_{\partial}$ induces a cohomology isomorphism

$$
\left(\mathcal{S}^{\mathrm{c}} \nabla\right)_{\partial}: \mathrm{H}^{*, *}\left(\operatorname{Hom}\left(\mathcal{S}_{[,, \cdot]}^{\mathrm{c}}\left[S^{-1}(L)\right], \mathbf{k}\right)\right) \rightarrow \mathrm{H}^{*, *}\left(\operatorname{Hom}\left(\mathcal{S}_{\mathcal{D}}^{\mathrm{c}}\left[S^{-1}(\mathrm{H}(L))\right], \mathbf{k}\right)\right) .
$$

Thus our coordinate ring $A\left(M_{L}\right)$ for $M_{L}$ now appears as the algebra $\mathrm{H}^{0,0}\left(\operatorname{Hom}\left(\mathcal{S}_{\mathcal{D}}^{\mathrm{c}}\left[S^{-1}(\mathrm{H}(L))\right], \mathbf{k}\right)\right)$. When we reinterpret this in terms of $\mathrm{H}(L)$, we find that, at least formally, $M_{L}$ may be written as the quotient $M_{L}=W_{L} / F$ of what is called the miniversal "variety" $W_{L} \subseteq \mathrm{H}^{1}(L)$ by an equivalence relation $F$. Here $W_{L}$ is defined by formal power series which are determined by the perturbation $\mathcal{D}$ and, likewise, the equivalence relation $F$ is determined by $\mathcal{D}$.

In particular, when $L$ is formal, that is, when $\tau$ and $\mathcal{D}$ can be constructed in such a way that $\mathcal{D}$ is merely given by the induced Lie bracket on $\mathrm{H}(L)$, then $W_{L}$ is the pure quadratic "variety" which consists of all $\eta \in \mathrm{H}^{1}(L)$ having the property that $[\eta, \eta]=0 \in \mathrm{H}^{2}(L)$. Under such circumstances if, in addition, $\mathbf{k}$ is the field of real numbers, the (infinitesimal) $\mathrm{H}^{0}(L)$-action on $W_{L}$ then determines a foliation, and the moduli space $M_{L}$ is the space of leaves; under appropriate circumstances, $M_{L}$ may in fact be written as the space of $\exp \left(\mathrm{H}^{0}(L)\right)$-orbits with respect to an induced action of the Lie group $\exp \left(\mathrm{H}^{0}(L)\right)$ on $W_{L}$.

Under the circumstances of Barannikov and Kontsevich [1], the induced bracket on $\mathrm{H}^{*}(L)$ is actually trivial whence the "thickened" moduli space (which initially is a subspace of $\mathrm{H}^{*}(L)$ ) is affine, in fact it is all of $\mathrm{H}^{*}(L)$; or for the Kodaira-Spencer algebra the moduli space would be just $\mathrm{H}^{1}(L)$.

Schlessinger-Stasheff [45] consider deformations of rational homotopy types which can be described also as deformations of connected graded commutative algebras with "symmetric" $A_{\infty}$-structures. Rather than reviewing that theory, here is a particular application which also provides a good example of how our result is more general than that of Barannikov and Kontsevich.

Consider the case of the cohomology of a wedge of spheres $X=\vee S^{n_{j}}$. Its cohomology algebra $\mathrm{H}^{*}$ with any field coefficients $\mathbf{k}$ is (in upper degrees) a nonnegatively graded vector space with $\mathrm{H}^{0}=\mathbf{k}$ and all products of positive degree 
elements being zero. The rational homotopy groups $\pi_{*}(\Omega X) \otimes \mathbb{Q}$ of the based loop space $\Omega X$ of $X$ are then isomorphic to $\mathcal{L}\left(s \overline{\mathrm{H}}_{*}(X)\right)$ [20], the free graded Lie algebra generated by the reduced homology $\overline{\mathrm{H}}_{*}(X)$ of $X$, shifted by 1 in grading. The deformation theory of rational homotopy types refers to the classification of rational homotopy types with the same cohomology algebra; the different types can, in this example, be distinguished by Massey products: higher order operations in cohomology. Attaching a cell by an ordinary Whitehead product $\left[S^{p}, S^{q}\right]$ means the cell carries the product cohomology class. We rule out this change in the algebra. Massey and Uehara [47,40] introduced Massey products in order to detect cells attached by iterated Whitehead products such as $\left[S^{p},\left[S^{q}, S^{r}\right]\right]$.

Now consider the differential graded Lie algebra $\operatorname{Coder}\left(\mathcal{L}^{\mathrm{c}}\left(S \overline{\mathrm{H}}^{*}(X)\right)\right)$ where $\mathcal{L}^{\mathrm{c}}\left(S^{-1} \overline{\mathrm{H}}^{*}(X)\right)$ denotes the free Lie coalgebra on the shifted cohomology $\overline{\mathrm{H}}^{*}(X)$. Since all products of positive degree elements are zero, we start with $d=0$ on $\mathcal{L}^{\mathrm{c}}\left(S \overline{\mathrm{H}}^{*}(X)\right)$. A perturbation $\theta$ (of the zero differential) decomposes into pieces $\theta_{k}$ which can be identified with homomorphisms

$$
\theta_{k}: \mathrm{H}^{\otimes(k+2)} \rightarrow \mathrm{H}
$$

Consider the case in which $\theta=\theta_{k}$ is homogeneous and has non-zero image in only one $\mathrm{H}^{n}$. The corresponding space $Y$ is obtained from $X$ by attaching the corresponding $n$-cells not as spheres in the bouquet but non-trivially according to the iterated Whitehead product determined by the pre-images under $\theta$. In this space, all Massey products of order less than $k+2$ will vanish but $\theta$ will represent a (sum of) non-trivial Massey product(s).

The differential graded Lie algebra $\pi_{*}(\Omega Y) \otimes \mathbb{Q}$ is no longer sh-equivalent to the homology of $\mathcal{L}\left(s \overline{\mathrm{H}}_{*}(X)\right)$ with trivial differential but rather to the homology with respect to the corresponding perturbation. In other words, this differential graded Lie algebra is not formal.

An example with "continuous moduli", i. e., of a one-parameter family of homotopy types, was first mentioned to us by J. Morgan: Let $X=S^{3} \vee S^{3} \vee S^{12}$, so that the space of possible 5-fold Massey products $\mathrm{H}^{\otimes 5} \rightarrow \mathrm{H}$ is of dimension 6 - equivalently, the attaching maps are in $\pi_{11}\left(S^{3} \vee S^{3}\right) \otimes \mathbb{Q}$ which is of dimension 6. A quick check of the relevant dimensions shows that there are no possible infinitesimal automorphisms, and $\operatorname{Aut}(\mathrm{H})=\mathrm{GL}(2) \times \mathrm{GL}(1)$ is of dimension 5; thus the Massey products distinguish at least a 1-parameter family.

\section{References}

1. S. Barannikov and M. Kontsevich, Frobenius manifolds and formality of Lie algebras of polyvector fields, alg-geom/9710032, Internat. Math. Res. Notices 4 (1998), 201-215.

2. I. A. Batalin and G. S. Vilkovisky, Quantization of gauge theories with linearly dependent generators, Phys. Rev. D 28 (1983), 2567-2582.

3. E. Brown, Twisted tensor products.I., Ann. of Math. 69 (1959), 223-246.

4. H. Cartan, Notions d'algèbre différentielle; applications aux groupes de Lie et aux variétés où opère un groupe de Lie, Bruxelles, Coll. Topologie Algébrique (1950), 15-28.

5. H. Cartan, La transgression dans un groupe de Lie et dans un espace fibré principal, Bruxelles, Coll. Topologie Algébrique (1950), 57-72. 
6. H. Cartan, Algèbres d'Eilenberg-Mac Lane et homotopie, exposés 2-11, Séminaire H. Cartan 1954/55, Ecole Normale Superieure, Paris, 1956.

7. H. Cartan and S. Eilenberg, Homological Algebra, Princeton University Press, Princeton, 1956.

8. K.T. Chen, Iterated path integrals, Bull. Amer. Math. Soc. 83 (1977), 831-879.

9. K. T. Chen, Extension of $C^{\infty}$ Function Algebra by Integrals and Malcev Completion of $\pi_{1}$, Advances in Mathematics 23 (1977), 181-210.

10. P. Deligne, P. Griffiths, J. Morgan, and D. Sullivan, Real homotopy theory of Kähler manifolds, Inv. Math. 29 (1975), 245-274.

11. S. Eilenberg and S. Mac Lane, On the groups $\mathrm{H}(\pi, n)$. I., Ann. of Math. 58 (1953), 55-106; II. Methods of computation, Ann. of Math. 60 (1954), 49-139.

12. M. Gerstenhaber and S. D. Schack, Algebras, bialgebras, quantum groups and algebraic deformations, In: Deformation theory and quantum groups, with applications to mathematical physics, M. Gerstenhaber and J. Stasheff, eds., Cont. Math. 134 (1992), AMS, Providence, 51-92.

13. E. Getzler, Batalin-Vilkovisky algebras and two-dimensional topological field theories, Comm. in Math. Phys. 195 (1994), 265-285.

14. V.K.A.M. Gugenheim, On the chain complex of a fibration, Illinois J. of Mathematics 16 (1972), 398-414.

15. V.K.A.M. Gugenheim, On a perturbation theory for the homology of the loop space, J. of Pure and Applied Algebra 25 (1982), 197-205.

16. V.K.A.M. Gugenheim and L. Lambe, Perturbation in differential homological algebra, Illinois J. of Mathematics 33 (1989), 566-582.

17. V.K.A.M. Gugenheim, L. Lambe, and J.D. Stasheff, Algebraic aspects of Chen's twisting cochains, Illinois J. of Math. 34 (1990), 485-502.

18. V.K.A.M. Gugenheim, L. Lambe, and J.D. Stasheff, Perturbation theory in differential homological algebra. II., Illinois J. of Math. 35 (1991), 357-373.

19. V.K.A.M. Gugenheim and H. J. Munkholm, On the extended functoriality of Tor and Cotor, J. of Pure and Applied Algebra 4 (1974), 9-29.

20. P. J. Hilton, On the homotopy groups of the union of spheres, J. of the London Math. Soc. 30 (1955), 154-172.

21. J. Huebschmann, The homotopy type of $F \Psi^{q}$. The complex and symplectic cases, in: Applications of Algebraic $K$-Theory to Algebraic Geometry and Number Theory, Part II, Proc. of a conf. at Boulder, Colorado, June 12 - 18, 1983, Cont. Math. 55 (1986), 487-518.

22. J. Huebschmann, Perturbation theory and free resolutions for nilpotent groups of class 2, J. of Algebra 126 (1989), 348-399.

23. J. Huebschmann, Cohomology of nilpotent groups of class 2, J. of Algebra 126 (1989), 400-450.

24. J. Huebschmann, The mod $p$ cohomology rings of metacyclic groups, J. of Pure and Applied Algebra 60 (1989), 53-105.

25. J. Huebschmann, Cohomology of metacyclic groups, Trans. Amer. Math. Soc. 328 (1991), 1-72.

26. J. Huebschmann, Extensions of Lie-Rinehart algebras and the Chern-Weil construction, in: Festschrift in honor of J. Stasheff's 60th birthday, Cont. Math. 227 (1999), Amer. Math. Soc., Providence R. I., 145-176. 
27. J. Huebschmann, Lie-Rinehart algebras, Gerstenhaber algebras, and BatalinVilkovisky algebras, Annales de l'Institut Fourier 48 (1998), 425-440.

28. J. Huebschmann, Rinehart complexes and Batalin-Vilkovisky algebras, preprint 2000.

29. J. Huebschmann, Twilled Lie-Rinehart algebras and differential Batalin-Vilkovisky algebras, math.DG/9811069.

30. J. Huebschmann, Differential Batalin-Vilkovisky algebras arising from twilled LieRinehart algebras, Poisson Geometry, Banach Center publications 51 (2000), 87-102.

31. J. Huebschmann, Berikashvili's functor $\mathcal{D}$ and the deformation equation, Festschrift in honor of N. Berikashvili's 70th birthday; math.AT/9906032, Proceedings of the A. Razmadze Mathematical Institute 119 (1999), 59-72.

32. J. Huebschmann and T. Kadeishvili, Small models for chain algebras, Math. Z. 207 (1991), 245-280.

33. M. Kodaira, L. Nirenberg and D. C. Spencer, On the existence of deformations of complex analytic structures, Ann. of Math. 68 (1958), 450-457.

34. Y. Kosmann-Schwarzbach, Exact Gerstenhaber algebras and Lie bialgebroids, Acta Applicandae Mathematicae 41 (1995), 153-165.

35. J. L. Koszul, Crochet de Schouten-Nijenhuis et cohomologie, in E. Cartan et les Mathématiciens d'aujourd'hui, Lyon, 25-29 Juin, 1984, Astérisque, hors-série, (1985), 251-271.

36. T. Lada and J. Stasheff, Introduction to sh Lie algebras for physicists, Int. J. Theor. Phys. 32 (1993), 1087-1104.

37. B. H. Lian and G. J. Zuckerman, New perspectives on the BRST-algebraic structure of string theory, Comm. in Math. Phys. 154 (1993), 613-646.

38. Yu. I. Manin, Frobenius manifolds, quantum cohomology, and moduli spaces, Colloquium Publications, vol. 47, Amer. Math. Soc., Providence, Rhode Island, 1999.

39. Yu. I. Manin, Three constructions of Frobenius manifolds: a comparative study, Sir Michael Atiyah: a great mathematician of the twentieth century, math.QA/9801006, Asian Math. J. 3 (1999), 179-220.

40. W. S. Massey, Some higher order cohomology operations, Universidad Nacional Autónoma de México and UNESCO, Mexico City, Symposium internacional dé topologia algebraica (1958), 145-154.

41. J. C. Moore, Differential homological algebra, Actes, Congrès intern. math. Nice (1970), Gauthiers-Villars, Paris, 1971, 335-339.

42. H. J. Munkholm, The Eilenberg-Moore spectral sequence and strongly homotopy multiplicative maps, J. of Pure and Applied Algebra 9 (1976), 1-50.

43. D. Quillen, Rational homotopy theory, Ann. of Math. 90 (1969), 205-295.

44. S. Saneblidze, Obstructions to the section problem in fibre bundles, manuscripta math. 81 (1993), 95-111.

45. M. Schlessinger and J. Stasheff, Deformation theory and rational homotopy type, to appear; new version July 13, 1998, Pub. Math. Sci. IHES.

46. M. Schlessinger and J. Stasheff, The Lie algebra structure of tangent deformation theory, J. of Pure and Applied Algebra 38 (1985), 313-322.

47. H. Uehara and W. S. Massey, The Jacobi identity for Whitehead products, Algebraic geometry and topology, a symposium in honor of S. Lefschetz (1957), 
361-377.

48. P. Xu, Gerstenhaber algebras and BV-algebras in Poisson geometry, Comm. in Math. Phys. 200 (1999), 545-560. 